\title{
Profiles of Biomarkers of Excess Alcohol Consumption in Patients Undergoing Total Hip Replacement: Correlation with Function
}

\author{
Paul J. Jenkins, ${ }^{1}$ Andrew D. Duckworth, ${ }^{2}$ Francis P. C. Robertson, ${ }^{2}$ \\ Colin R. Howie, ${ }^{2}$ and James S. Huntley ${ }^{3}$ \\ ${ }^{1}$ Division of Clinical and Surgical Sciences, Department of Orthopaedics, University of \\ Edinburgh, Room FU413, Chancellor's Building, Royal Infirmary of Edinburgh, \\ Old Dalkeith Road, Edinburgh EH16 4SB, UK \\ ${ }^{2}$ Department of Orthopaedic and Trauma Surgery, Royal Infirmary of Edinburgh, \\ Old Dalkeith Road, Edinburgh EH16 4SA, UK \\ ${ }^{3}$ Department of Orthopaedics, Royal Hospital for Sick Children, Yorkhill, Dalnair Street, \\ Glasgow G3 8SJ, UK
}

Received 25 July 2011; Accepted 23 August 2011

Academic Editor: Robin Touquet

\begin{abstract}
Aims. Patients who misuse alcohol may be at increased risk of surgical complications and poorer function following hip replacement. Identification and intervention may lead to harm reduction and improve the outcomes of surgery. The aim of this study was to determine the prevalence of biomarker elevation in patients undergoing hip replacement and to investigate any correlation with functional scores and complications. Methods. We performed a retrospective study that examined the profile of biomarkers of alcohol misuse in 1049 patients undergoing hip replacement. Results. Gamma-glutamyltransferase was elevated in 150 (17.6\%), and mean corpuscular volume was elevated in $23(4 \%)$. At one year general physical health was poorer where there was elevation of $\gamma \mathrm{GT}$, and the mental health and hip function was poorer with elevation of MCV. There were no differences in complications. Discussion. Raised biomarkers can alert clinicians to potential problems. They also provide an opportunity to perform further investigation and offer intervention. Future research should focus on the use in orthopaedic practice of validated screening questionnaires and more sensitive biomarkers of alcohol misuse. Conclusion. This study demonstrates a potential substantial proportion of unrecognised alcohol misuse that is associated with poorer functional scores in patients after total hip replacement.
\end{abstract}

KEYWORDS: Total hip replacement, total hip arthroplasty, hip replacement, alcohol misuse, patient reported outcome measure 


\section{INTRODUCTION}

Excess alcohol consumption confers an increased risk of morbidity and mortality in patients undergoing surgical procedures [1,2]. Detection of alcohol misuse and intervention is effective and cost effective at reducing alcohol-related morbidity in the emergency department [3, 4]. Although the effect of alcohol misuse has been investigated in orthopaedic patients [5], there is little evidence about the prevalence of misuse in patients undergoing joint replacement and its effect on postoperative function and complications $[6,7]$. Most studies of outcome following total hip replacement do not report alcohol misuse, indeed a recent meta-analysis of patient characteristics affecting the prognosis of hip and knee joint arthroplasty did not report preoperative alcohol consumption [8].

Alcohol-related morbidity is a major problem-in the Scottish population there were 42.430 discharges from hospital with an alcohol-related diagnosis in 2007/8, equating to a rate of 777 per 100.000 [9]. Thirty-four percent of men and $23 \%$ of women are reported to exceed safe weekly drinking levels [10]. This has resulted in several reports by government, charity, and medical organisations targeting the causes, identification, and treatment of alcohol misuse [11-14]. Improved access to alcohol health workers has been advocated [15]. Treating the consequences of alcohol misuse costs the National Health Service billions of pound per year $[16,17]$. Multicriteria decision analysis has determined that alcohol is the most harmful substance of misuse [18].

Biomarkers can be used to identify patients with increased alcohol intake [19]. Though nonspecific, mean corpuscular volume (MCV) and gamma-glutamyl-transferase $(\gamma \mathrm{GT})$ are the most commonly used in clinical practice and are sensitive in the assessment of patients with high-risk drinking behaviours [20]. These are commonly available as part of routine haematological and biochemical tests at the time of surgery. More specific biomarkers do exists (carbohydrate-deficient transferrin: CDT); however, these tend to be used in limited clinical and research situations.

The aim of this study was to describe the prevalence of elevated biomarkers in patients undergoing hip arthroplasty. Secondary aims were to investigate whether biomarker elevation was correlated to (i) functional scores (preoperative, and at one year) and (ii) the development of complications.

\section{PATIENTS AND METHODS}

At our institution, the demographic details, general health status, and specific joint patient reported function were recorded in a prospectively collected audit database. From January 2005 to December 2006 (24 months), 1049 primary total hip replacements (THR) were performed. Patients attended a preassessment clinic two weeks prior to surgery for medical assessment, blood tests (full blood count, urea and electrolytes, and liver function), the SF-12 general health questionnaire [21], and the Oxford hip score [22]. The SF12 provides a mental and physical component summary that is normalised to the national population. The Oxford hip score measures disability in 12 domains on a Likert scale, with 12 representing the least disability and 60 representing the most disability. We excluded patients undergoing arthroplasty for rheumatoid arthritis $(n=22)$, a second procedure on the contralateral hip within the study period $(n=44)$, and those with no preoperative functional score $(n=132)$. Therefore, 851 patients undergoing primary THR were included in the study group. There were 510 females (60\%) and 341 males (40\%). The mean age at surgery was 66.3 years (S.D. 12.2), and the mean followup was 58 months $(\mathrm{SD}=6.6)$. No patient had a concomitant diagnosis of pernicious anaemia to account for observed macrocytosis.

We retrospectively reviewed the computerised laboratory database to determine the preoperative $\gamma \mathrm{GT}$ and MCV. $\gamma \mathrm{GT}$ was determined by a kinetic colour test on an OSR6020 analyser (Olympus, Diagnostica, Ireland). The MCV was determined by an automated analyser (XE 2100, Sysmex UK, Wymbush, UK). CDT was not retrospectively available.

THR was performed by, or under the supervision of, one of fifteen consultant orthopaedic surgeons. Procedures were performed through either the modified Hardinge or posterior approach. The prostheses used during the study period were the cemented Exeter stem (Stryker, Newbury, UK) or Olympia (Biomet 


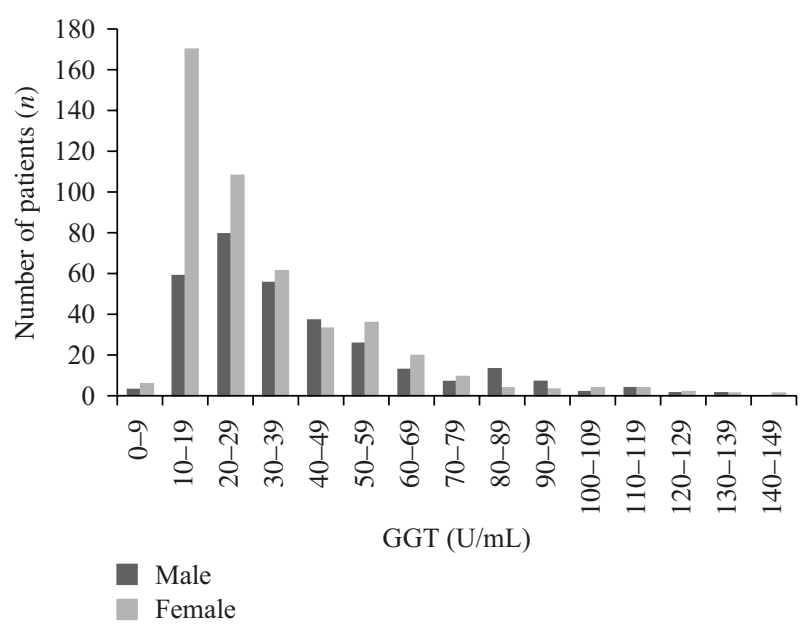

FIGURE 1: Distribution of patients undergoing total hip replacement by $\gamma$ GT and gender.

UK, Bridgend, UK) with a cemented cup. The local laboratory upper reference limit of normal (ULN) values was used to determine if elevation was present. This limit is set at two standard deviations above the healthy population mean thus identifying $95 \%$ of the healthy population as below this level. The cutoff for $\gamma$ GT in male patients was 55 U/L and in females was $35 \mathrm{U} / \mathrm{L}$. The ULN for the MCV was $99 \mathrm{fl}$ (femtolitres).

Data were obtained from ISD (Information and Statistics, Edinburgh, UK) and the Scottish Arthroplasty Project [23] to identify complications resulting in readmission to our unit, or any other hospital in Scotland. We identified thromboembolic episodes or deaths with 90 days of surgery. We also identified any dislocations, infection requiring readmission, or revision within one year of surgery.

Data were analysed using SPSS version 13 (Statistical Software for Social Sciences, Chicago, IL, USA). Normally distributed data were reported using means and standard deviations (SD), while nonnormal continuous data were reported with medians and interquartile ranges (IQRs). The Chi-squared test was used to determine the significance of differences between categorical data of elevated biomarkers and gender and complications. Functional outcomes were assessed for normality, and parametric tests were, therefore, used. Student $t$-tests were used to compare the mean preoperative and postoperative functional scores between two groups on the basis of biomarker elevation.

\section{RESULTS}

The median $\gamma$ GT was $28 \mathrm{U} / \mathrm{L}$ (IQR 18 to 47) and the median MCV was $91 \mathrm{fl}$ (IQR 88 to 94). There were $150(17.6 \%)$ patients with a $\gamma$ GT higher than the ULN. There were more males $(n=71,20.8 \%)$ with an elevated $\gamma$ GT than females $(n=79,15.4 \%)\left(P=0.047, \chi^{2}\right)$ (Figure 1). There were $34(4 \%)$ patients with elevated MCV (Figure 2). There was no difference in the proportion of males $(n=14,4.1 \%)$ and females $(n=20,3.9 \%)$ with elevated $\operatorname{MCV}\left(P=0.893, \chi^{2}\right)$. There were 10 patients $(1.2 \%)$ with combined elevation of both markers. The mean number of platelets was 264 (S.D. 75.1), and there was no statistical difference in platelet numbers between those with elevated $\gamma \mathrm{GT}(P=0.668, t$-test $)$ or $\mathrm{MCV}(P=0.408$, $t$-test).

Two hundred and fifty eight $(30.3 \%)$ patients did not complete one-year functional followup. A higher number of patients with an elevated MCV $(n=20 / 34,58.8 \%)$ failed to complete 1-year functional assessment than those within the "normal" limits $(n=238 / 817,29.1 \%)\left(P<0.001, \chi^{2}\right)$. There was no difference in loss to followup of patients with an elevated $\gamma \mathrm{GT}(n=49 / 150,32.7 \%)$ compared with patients with $\gamma$ GT below the ULN $(n=209 / 701,29.8 \%)$. 


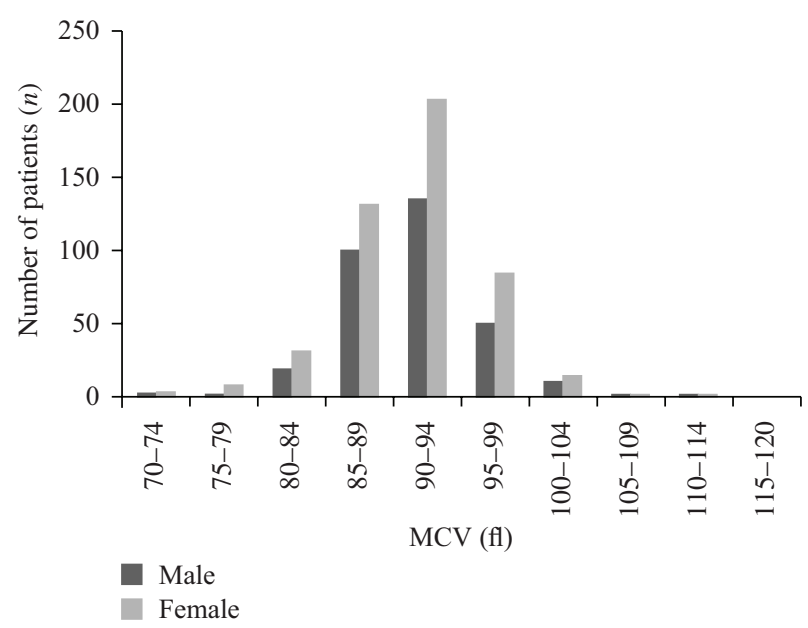

FIGURE 2: Distribution of patients undergoing total hip replacement by MCV and gender.

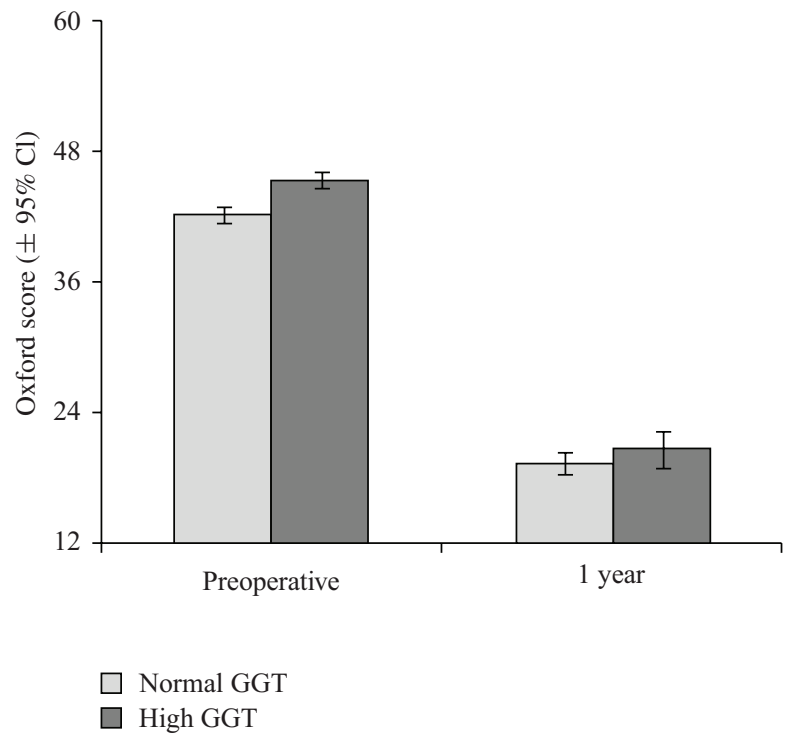

FIGURE 3: Preoperative and 1 Year Oxford Hip Score in patients with normal and raised $\gamma$ GT.

At preoperative assessment, the Oxford Hip Score (OHS) was significantly higher in patients with elevation of either $\gamma \mathrm{GT}(P=0.007)$ or $\mathrm{MCV}(P=0.010)$ (Figure 3). Raised $\gamma \mathrm{GT}$ or MCV was not associated with derangements of either the physical or mental component scores of the SF-12 score (Tables 1 and 2).

The Oxford score had improved by 1 year in all patients (mean improvement $=20.0,95 \%$ CI 19.2 to 20.9, $P<0.001$ ), as had both the physical (mean improvement $=12.2,95 \%$ CI 11.3 to $13.2, P<0.001$ ) and mental components (mean improvement $=3.7,95 \%$ CI 2.8 to $4.7, P<0.001$ ) of the SF-12 score. There were no significant differences in any of the changes in scores between those with either marker elevation (Tables 1 and 2).

At one-year assessment, there was a significantly poorer physical SF-12 score among those patients with elevated preoperative $\gamma$ GT. In those with an elevation of MCV, there were significantly poorer SF-12 mental scores $(P=0.026)$ and Oxford hip scores $(P=0.039)$ (Table 2$)$. 
TABLE 1: Mean Functional Scores at preoperative assessment in patients with normal and elevated biomarker.

\begin{tabular}{lcccccc}
\hline Functional score (Mean, SD) & \multicolumn{3}{c}{$\gamma$ GT } & \multicolumn{3}{c}{ MCV } \\
& Elevated & Normal & $P$ value & Elevated & Normal & $P$ value \\
\hline SF-12 & & & & & & \\
$\quad$ Physical & $28.6(6.6)$ & $29.2(7.1)$ & 0.343 & $27.9(7.2)$ & $29.1(7.0)$ & 0.294 \\
$\quad$ Mental & $46.1(12.1)$ & $48.2(12.0)$ & 0.060 & $44.7(11.8)$ & $47.9(12.1)$ & 0.129 \\
Oxford Hip Score & $44.8(7.1)$ & $42.8(8.1)$ & $\mathbf{0 . 0 0 7}$ & $46.6(7.1)$ & $43.0(7.9)$ & $\mathbf{0 . 0 1 0}$ \\
\hline
\end{tabular}

TABLE 2: Mean function scores at 1 year assessment in patients with normal and elevated biomarkers.

\begin{tabular}{|c|c|c|c|c|c|c|}
\hline \multirow{2}{*}{ Functional score (Mean, SD) } & \multicolumn{3}{|c|}{$\gamma \mathrm{GT}$} & \multicolumn{3}{|c|}{$\mathrm{MCV}$} \\
\hline & Elevated & Normal & $P$ value & Elevated & Normal & $P$ value \\
\hline \multicolumn{7}{|l|}{ SF-12 } \\
\hline Physical & $39.6(11.4)$ & $42.2(11.1)$ & 0.036 & $37.6(11.5)$ & $41.8(11.2)$ & 0.165 \\
\hline Mental & $51.5(11.5)$ & $53.0(10.2)$ & 0.201 & $46.5(12.6)$ & $52.8(10.4)$ & 0.026 \\
\hline Oxford Hip Score & $23.7(10.5)$ & $22.4(9.5)$ & 0.231 & $27.9(12.5)$ & $22.5(9.5)$ & 0.039 \\
\hline
\end{tabular}

TABLE 3: Complications in patients with normal and elevated biomarkers.

\begin{tabular}{lcccc}
\hline \multirow{2}{*}{ Complication } & \multicolumn{2}{c}{$\gamma$ GT } & \multicolumn{2}{c}{ MCV } \\
& Normal $(n=701)$ & Elevated $(n=150)$ & Normal $(n=817)$ & Elevated $(n=34)$ \\
\hline DVT/PE $(n=12)$ & $9(1.3 \%)$ & $3(2 \%)$ & $11(1.3 \%)$ & $1(2.9 \%)$ \\
Infection $(n=6)$ & $5(0.7 \%)$ & $1(0.7 \%)$ & $6(0.7 \%)$ & 0 \\
Dislocation $(n=1)$ & 0 & $1(0.7 \%)$ & $1(0.1 \%)$ & 0 \\
Fracture $(n=2)$ & $2(0.3 \%)$ & 0 & $2(0.2 \%)$ & 0 \\
Revision $(n=9)$ & $8(1.1 \%)$ & $1(0.7 \%)$ & $8(1.0 \%)$ & $1(2.9 \%)$ \\
Death & $2(0.3 \%)$ & 0 & $1(0.1 \%)$ & $1(2.9 \%)$ \\
\hline
\end{tabular}
(Table 3).

Raised preoperative biomarkers were not associated with a difference in postoperative complications

\section{DISCUSSION}

This study demonstrates a substantial proportion of unrecognised alcohol misuse in those undergoing total hip replacement, which is similar to the reported national proportion of hazardous drinking [9]. It further suggests that these patients are less likely to comply with standard functional followup. It also highlights increased disability among patients with elevated biomarkers (1) before their operation and (2) poorer physical and mental outcomes at 1 year. There was no significant difference (between groups of patients with raised biomarkers versus those with normal biomarkers) in the absolute improvement in any functional score or rate of complications.

The MCV may be elevated in these patients due to deficiency of vitamin B compounds. $\gamma \mathrm{GT}$ is a liver enzyme involved in transport of amino-acids across cell membranes. It is released into the blood stream by cellular damage. Several studies have used these biomarkers, either in isolation or in combination with questionnaire tools. The sensitivity and specificity of $\gamma$ GT to detect hazardous drinking (when compared with the CAGE questionnaire) were 0.69 and 0.82 , respectively, whereas the sensitivity of MCV is lower 
at 0.27 , but is more specific (0.91) than $\gamma$ GT [24]. The literature on MCV is contradictory; another study reported a higher sensitivity for MCV of 0.70 [25]. Biomarkers have been used in various populations to detect hazardous drinking [26]. $\gamma$ GT may be elevated by other medication or cholestasis and MCV may be elevated by other conditions causing macrocytic anaemia such as pernicious anaemia. Carbohydrate deficient transferrin (CDT) is a biomarker of excess alcohol consumption that has shown a more favourable test profile with a reported sensitivity of 0.58 and specificity of 0.82 [24]. It is the increased specificity that may lead to more accurate detection of alcohol misuse [26].

Alcohol misuse is very common in the UK population and patients may possess "hazardous drinking" patterns that increase their risk of harmful consequences. "Hazardous drinking" may lead to "harmful drinking" where there is actual physical and/or mental impairment [27]. Such behaviour may lead to alcohol dependence syndromes. The most recent UK government guidelines advise men should not regularly drink more than three to four units ( 1 unit $=7.9 \mathrm{~g}$ of ethanol per $100 \mathrm{~mL}$ ) of alcohol per day and women no than two to three units. In the course of a week, men are advised to drink no more than 21 units per week, and women no more than 14 units. Patterns of "binge drinking" are observed where there is episodic ingestion of more than twice the advised daily limits [28]. The definition of a unit of alcohol varies between countries, as do the sensible drinking guidelines [29].

Harmful drinking may result in perioperative dysrhythmia [30], immune response impairment [31], and coagulation disorders. Wound healing and surgical site infection have been ascribed to suppression of collagen formation [32]. There is a high risk of postoperative delirium in those with dependence and withdrawal syndromes [33]. The stress response to surgery, mediated by the hypothalamo-pituitary axis, is also potentiated in alcohol misuse [34]. A clinical effect of the rate of complications has been detected in those with alcohol misuse undergoing joint replacement [5]. Alcohol misuse may also lead to the development of secondary degenerative joint disease through osteonecrosis [7, 35].

Alcohol intake and misuse can be reliably identified by validated questionnaires [36]. The PAT tool has been used effectively in the emergency department setting to rapidly identify harmful drinking and direct brief interventions by an alcohol health worker (AHW) [3, 37]. The FAST [38], CAGE [39], and RAPS4 [40] questionnaires are similar tools that would be appropriate for screening for harmful drinking at preoperative assessment. The delivery of brief interventions has been shown to be cost effective [3].

The main limitations of this study are its retrospective nature and rate of loss to followup, which was higher among patients with elevated biomarkers of alcohol misuse. This may lead to an error in estimation of function in this group. Our study is also limited by the sensitivity and specificity of the biomarkers. Although there were no differences detected in the rate of complications (a secondary research question), the low incidence of such events in either group may require a larger sample size to detect a statistically significant difference. Also, surgical strategies to deal with suspected alcohol misuse confound the measurement of an effect. The use of the CDT biomarker would have been desirable but the study was limited by the retrospective availability of $\gamma \mathrm{GT}$ and $\mathrm{MCV}$ as standard biomarkers obtained at preoperative assessment.

These limitations encourage us to advocate prospective evaluation of patients undergoing arthroplasty with validated alcohol use questionnaires preoperatively and a more sensitive biomarker assay. Delivery of brief interventions in secondary care may reduce alcohol misuse and, therefore, reduce hazardous and harmful drinking, leading to improved general health and risk of complications. Orthopaedic surgeons should be aware of local referral options for intervention in patients with hazardous drinking.

\section{CONFLICT OF INTERESTS}

The authors declare that they do not have any conflict interests or financial interests pertaining to this paper.

\section{ACKNOWLEDGMENTS}

The authors would like to thank Mr. Tim Norwood, Senior Analyst, ISD, NHS National Services Division and Mrs. Deborah MacDonald, Department of Orthopaedics, University of Edinburgh. 


\section{REFERENCES}

[1] H. Tønnesen and H. Kehlet, "Preoperative alcoholism and postoperative morbidity," British Journal of Surgery, vol. 86, no. 7, pp. 869-874, 1999.

[2] H. Tonnesen, A. Pedersen, M. R. Jensen, A. Moller, and J. C. Madsen, "Ankle fractures and alcoholism. The influence of alcoholism on morbidity after malleolar fractures," The Journal of Bone and Joint Surgery. British, vol. 73, no. 3, pp. 511-513, 1991.

[3] B. Barrett, S. Byford, M. J. Crawford et al., "Cost-effectiveness of screening and referral to an alcohol health worker in alcohol misusing patients attending an accident and emergency department: a decision-making approach," Drug \& Alcohol Dependence, vol. 81, no. 1, pp. 47-54, 2006.

[4] M. J. Crawford, R. Patton, R. Touquet et al., "Screening and referral for brief intervention of alcohol-misusing patients in an emergency department: a pragmatic randomised controlled trial," The Lancet, vol. 364, no. 9442, pp. 1334-1339, 2004.

[5] A. H. S. Harris, R. Reeder, L. Ellerbe, K. A. Bradley, A. D. Rubinsky, and N. J. Giori, "Preoperative alcohol screening scores: association with complications in men undergoing total joint arthroplasty," The Journal of Bone and Joint Surgery. American, vol. 93, no. 4, pp. 321-327, 2011.

[6] G. Williams, M. Daly, E. M. Proude et al., "The influence of alcohol and tobacco use in orthopaedic inpatients on complications of surgery," Drug and Alcohol Review, vol. 27, no. 1, pp. 55-64, 2008.

[7] J. S. Huntley and R. Touquet, "Surgical Problems," in ABC of Alcohol, A. Paton and R. Touquet, Eds., Blackwell Publishing, BMJ Books, London, UK, 4th edition, 2005.

[8] P. L. Santaguida, G. A. Hawker, P. L. Hudak et al., "Patient characteristics affecting the prognosis of total hip and knee joint arthroplasty: a systematic review," Canadian Journal of Surgery, vol. 51, no. 6, pp. 428-436, 2008.

[9] ISD Scotland, “Alcohol Statistics Scotland 2009," NHS National Services, Edinburgh, UK, 2009.

[10] The Scottish Government, "Scottish Health Survey: Revised Alcohol Consumption Estimates 2003," The Scottish Goverment, Edinburgh, UK, 2008.

[11] House of Commons Health Committee, “Alcohol,” London, UK, January 2010.

[12] "Signs for improvement—commissioning interventions to reduce alcohol-related harm," Government Department of Health, London UK, 2010.

[13] "Alcohol misuse: tackling the UK epidemic," British Medical Association, London, UK, February 2008.

[14] Alcohol Concern, "Making alcohol a health priority: opportunities to reduce alcohol harms and rising costs," Alcohol Concern, London, UK, January 2011.

[15] K. J. Moriarty, H. Platt, S. Crompton et al., "Collaborative care for alcohol-related liver disease," Clinical Medicine, Journal of the Royal College of Physicians of London, vol. 7, no. 2, pp. 125-128, 2007.

[16] The NHS Confereration, "Too much of the hard stuff: what alcohol costs the NHS,” London, UK, January 2010.

[17] Scottish Government, "Costs of use and misuse of alcohol in Scotland," 2008, http://www.scotland.gov.uk/ Publications/2008/05/06091510/0.

[18] D. J. Nutt, L. A. King, and L. D. Phillips, “Drug harms in the UK: a multicriteria decision analysis,” The Lancet, vol. 376, no. 9752, pp. 1558-1565, 2010.

[19] P. Sillanaukee, "Laboratory markers of alcohol abuse," Alcohol \& Alcoholism, vol. 31, no. 6, pp. 613-616, 1996.

[20] K. M. Conigrave, L. J. Degenhardt, J. B. Whitfield, J. B. Saunders, A. Helander, and B. Tabakoff, "CDT, GGT, and AST as markers of alcohol use: the WHO/ISBRA collaborative project," Alcoholism: Clinical and Experimental Research, vol. 26, no. 3, pp. 332-339, 2002.

[21] J. E. Ware, M. Kosinski, and S. D. Keller, "A 12-Item Short-Form Health Survey: Construction of scales and preliminary tests of reliability and validity," Medical Care, vol. 34, no. 3, pp. 220-233, 1996.

[22] J. Dawson, R. Fitzpatrick, A. Carr, and D. Murray, "Questionnaire on the perceptions of patients about total hip replacement," The Journal of Bone and Joint Surgery. British, vol. 78, no. 2, pp. 185-190, 1996.

[23] "Scottish Arthroplasty Project Report 2008," 2008, http://www.arthro.scot.nhs.uk/Reports/Scottish_Arthroplasty. Project_Report_2008.pdf.

[24] B. Yersin, J. F. Nicolet, H. Decrey, M. Burnier, G. Van Melle, and A. Pecoud, "Screening for excessive alcohol drinking: comparative value of carbohydrate-deficient transferrin, $\gamma$-glutamyltransferase, and mean corpuscular volume," Archives of Internal Medicine, vol. 155, no. 17, pp. 1907-1911, 1995. 
[25] H. Bell, C. Tallaksen, T. Sjaheim et al., "Serum carbohydrate-deficient transferrin as a marker of alcohol consumption in patients with chronic liver diseases," Alcoholism: Clinical and Experimental Research, vol. 17, no. 2, pp. 246-252, 1993.

[26] B. Hock, M. Schwarz, I. Domke et al., "Validity of carbohydrate-deficient transferrin (\%CDT), $\gamma$ glutamyltransferase $(\gamma-\mathrm{GT})$ and mean corpuscular erythrocyte volume (MCV) as biomarkers for chronic alcohol abuse: a study in patients with alcohol dependence and liver disorders of non-alcoholic and alcoholic origin," Addiction, vol. 100, no. 10, pp. 1477-1486, 2005.

[27] "Lexicon of alcohol and drug terms," 2008, http://www.who.int/.

[28] Prime Minister's Strategy Unit, "Interim analytical report for the national alcohol harm reduction strategy," Prime Minister's Strategy Unit; 2003.

[29] "Alcohol in Moderation. Sensible Drinking Guidelines," http://www.drinkingandyou.com/site/pdf/SENSIBLE\% 2520DRINKING.pdf.

[30] H. Tønnesen, “Alcohol abuse and postoperative morbidity," Danish Medical Bulletin, vol. 50, no. 2, pp. 139-160, 2003.

[31] L. La Vecchia, F. Bedogni, L. Bozzola, P. Bevilacqua, R. Ometto, and M. Vincenzi, "Prediction of recovery after abstinence in alcoholic cardiomyopathy: role of hemodynamic and morphometric parameters," Clinical Cardiology, vol. 19, no. 1, pp. 45-50, 1996.

[32] L. N. Jorgensen, F. Kallehave, T. Karlsmark, and F. Gottrup, "Reduced collagen accumulation after major surgery," British Journal of Surgery, vol. 83, no. 11, pp. 1591-1594, 1996.

[33] J. A. Hudetz, Z. Iqbal, S. D. Gandhi et al., "Postoperative cognitive dysfunction in older patients with a history of alcohol abuse," Anesthesiology, vol. 106, no. 3, pp. 423-430, 2007.

[34] K. Ogilvie, S. Lee, and C. Rivier, "Effect of three different modes of alcohol administration on the activity of the rat hypothalamic-pituitary-adrenal axis," Alcoholism: Clinical and Experimental Research, vol. 21, no. 3, pp. 467-476, 1997.

[35] L. C. Jones and D. S. Hungerford, "Osteonecrosis: etiology, diagnosis, and treatment," Current Opinion in Rheumatology, vol. 16, no. 4, pp. 443-449, 2004.

[36] B. Aertgeerts, F. Buntinx, S. Ansoms, and J. Fevery, "Screening properties of questionnaires and laboratory tests for the detection of alcohol abuse or dependence in a general practice population," British Journal of General Practice, vol. 51, no. 464, pp. 206-217, 2001.

[37] R. Touquet and A. Brown, "PAT (2009)—revisions to the paddington alcohol test for early identification of alcohol misuse and brief advice to reduce emergency department re-attendance," Alcohol \& Alcoholism, vol. 44, no. 3, pp. 284-286, 2009.

[38] R. J. Hodgson, B. John, T. Abbasi et al., "Fast screening for alcohol misuse," Addictive Behaviors, vol. 28, no. 8, pp. 1453-1463, 2003.

[39] J. A. Ewing, "Detecting alcoholism. The CAGE questionnaire," Journal of the American Medical Association, vol. 252, no. 14, pp. 1905-1907, 1984.

[40] C. J. Cherpitel, "A brief screening instrument for problem drinking in the emergency room: the RAPS4," Journal of Studies on Alcohol, vol. 61, no. 3, pp. 447-449, 2000.

\section{This article should be cited as follows:}

Paul J. Jenkins, Andrew D. Duckworth, Francis P. C. Robertson, Colin R. Howie, and James S. Huntley, "Profiles of Biomarkers of Excess Alcohol Consumption in Patients Undergoing Total Hip Replacement: Correlation with Function," TheScientificWorldJOURNAL, vol. 11, pp. 1804-1811, 2011. 


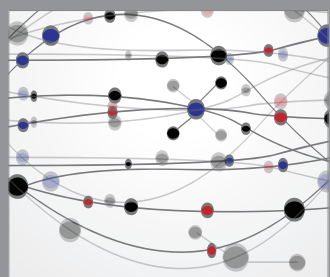

The Scientific World Journal
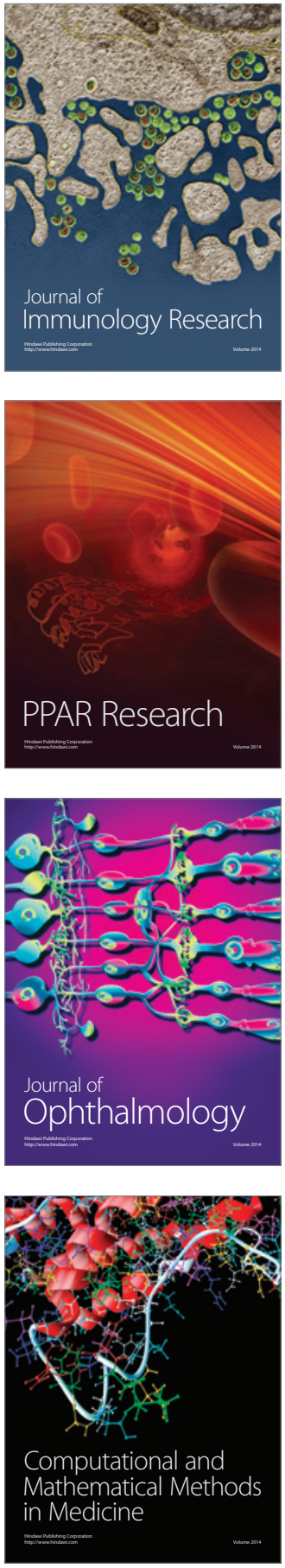

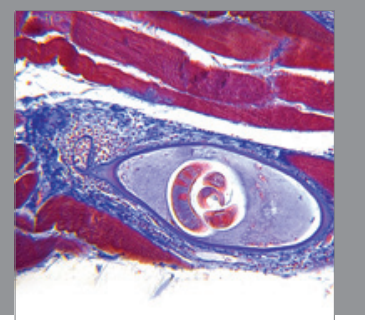

Gastroenterology

Research and Practice
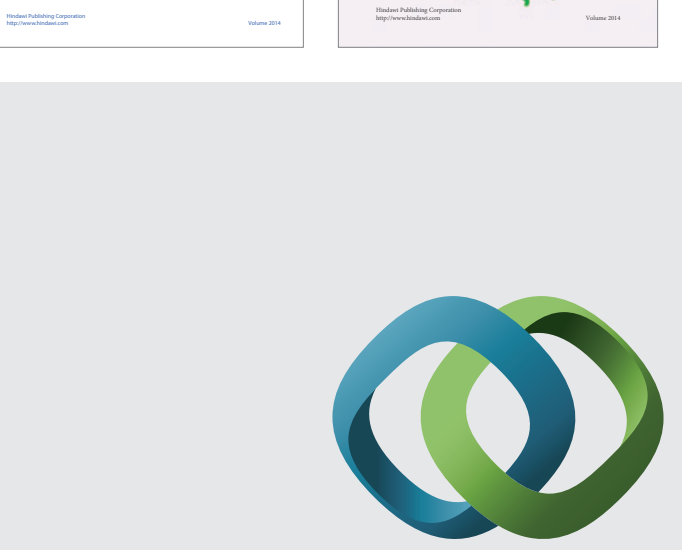

\section{Hindawi}

Submit your manuscripts at

http://www.hindawi.com
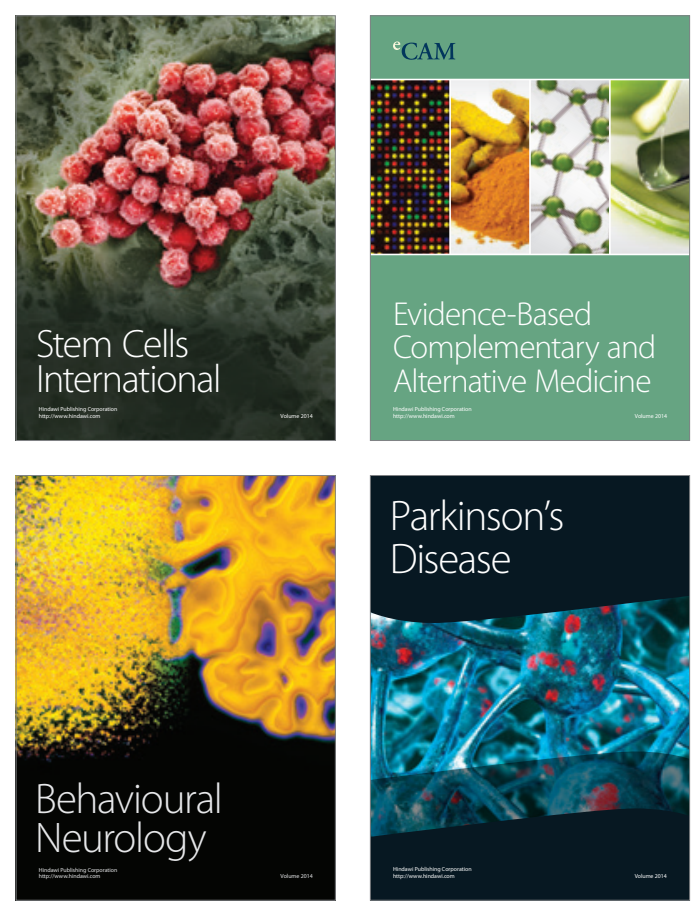

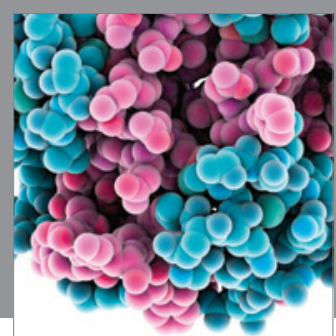

Journal of
Diabetes Research

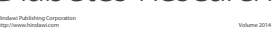

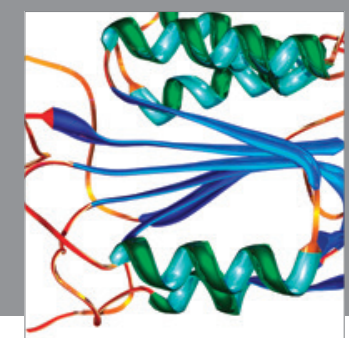

Disease Markers
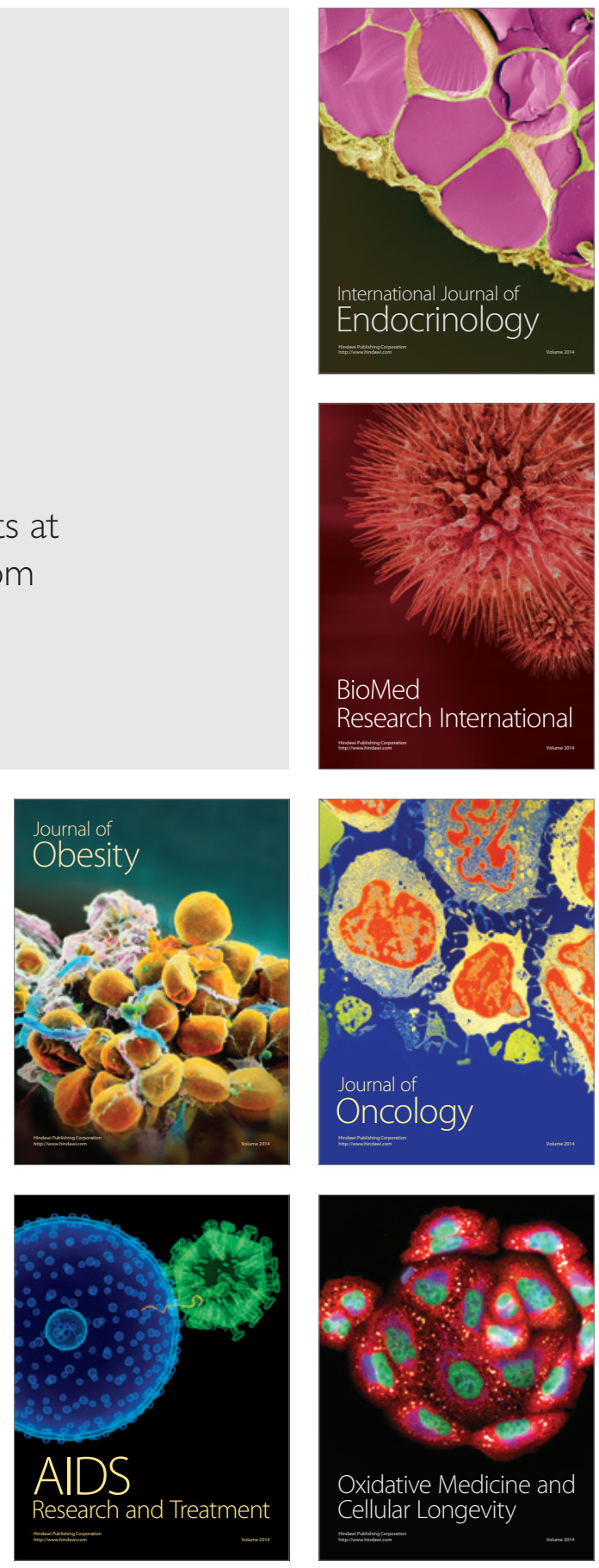